\title{
Scotland launches clinical effectiveness initiative
}

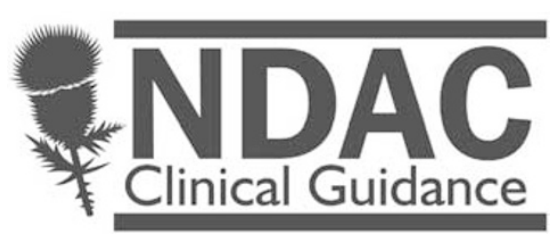

\author{
National Dental Advisory Committee-Clinical Effectiveness Pro- \\ gramme logo.
}

Scotland has recently established the Clinical Effectiveness Programme with funding from the Scottish Executive. The programme has been set up under the auspices of the National Dental Advisory Committee (NDAC), a body of dental staff representing all branches of the profession, which acts in an advisory capacity to the Chief Dental Officer. It considers issues that are of national importance in Scottish dentistry and also provides feedback to other groups within the Scottish Executive on related healthcare matters. Periodically, subgroups of this committee have produced publications, for example, Emergency Dental Drugs, Clinical Governance in Dental Primary Care and The Role of Dental Practice Advisors.

The primary aim of this new programme is to provide userfriendly, evidence-based guidance for dentists in Scotland. It also presents an opportunity to develop not only guidelines but also other mechanisms to support dentists' clinical and organisational decision making.

For the initial phase of the programme, NDAC has identified seven priority areas for production of guidance documents (Table 1). For each subject area, an individual with specific expertise and a national reputation in the field has been selected to chair a working group, which will undertake the guidance development process. In recognition of the fact that most dental treatment is provided in primary care, NDAC has insisted that the membership of each working group must include a significant proportion of primary care practitioners and professionals complementary to dentistry (PCD) staff. Thus, each working group includes general dental practitioners, community dentists, PCDs and academics, to ensure that all stakeholders have a voice in the development process. Working groups will be supported by the Guidance Support Team, with the process being overseen by a steering committee.

The methodology for guidance development will adhere as far as possible to internationally recognised standards set out by the AGREE Collaboration (www.agreecollaboration.org). To maximise

Table 1. NDAC priority areas.

\section{Area}

Care pathways for children with dental disease

Care pathways for emergency dental care

Decontamination and infection control

Dental prescribing

Dental sedation

Initial examination and assessment of patients

Practice support manual the impact of the guidance produced, the programme will be using dissemination and implementation methods that are themselves based on available evidence. With this in mind, the programme is supported by National Health Service, Education for Scotland (NES), and the three Scottish dental institutions, which will facilitate the integration of new guidance into education and training. Given the size and relative autonomy of the dental profession within Scotland, this is a realistic opportunity to implement guidance in a supportive manner and then to evaluate its effectiveness, thus informing the best way to facilitate changes in practice in the future.

For further information about any aspect of the programme, contact the administrator, Emma Hart (e.hart@dundee. ac.uk), or the senior researcher, Doug Stirling (d.a.stirling@dundee. ac.uk).

\section{Centre for Evidence-based Dentistry launches major overhaul of website}

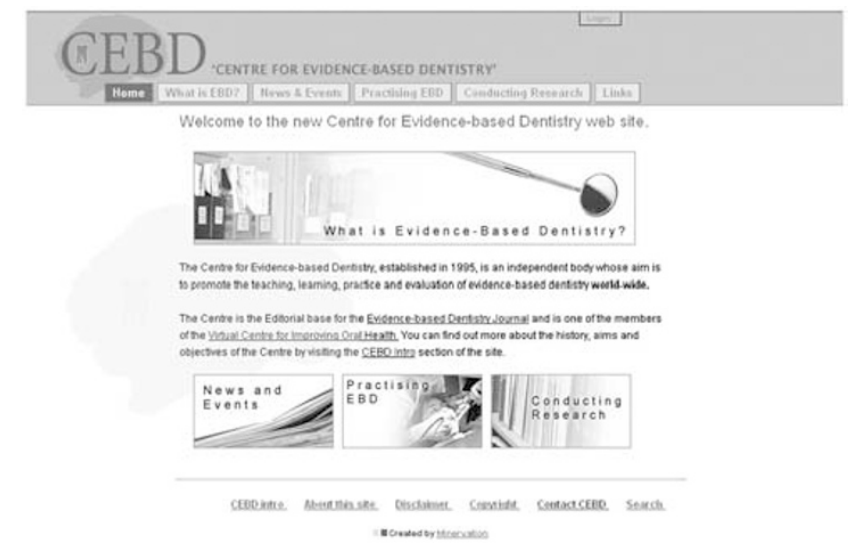

Centre for Evidence-based Dentistry home page.

At the recent International Association for Dental Research meeting in Baltimore the Centre for Evidence-based Dentistry (CEBD) announced the launch of its new and updated website (www.cebd. org). The new website has been developed by the CEBD working with the Oxford-based company Minervation (minervation.com). The site now provides clearer navigation and new sections on how to practice evidence-based dentistry and conducting research. We will be having a more detailed look at the site in a forthcoming issue.

Evidence-based Dentistry Network meeting in Baltimore The recent International Dental Research (IADR) meeting in the US, in Baltimore, Maryland, saw the first formal meeting of the Evidence-based Dentistry Network (EBNet).

EBNet has been in development over the past few years before its formal acknowledgement by IADR in 2004. It jointly sponsored an introductory workshop on meta-analysis and a symposium on systematic reviews in dental and craniofacial research. Both events were presented by high-quality speakers and were well attended, as was the inaugural network meeting. 


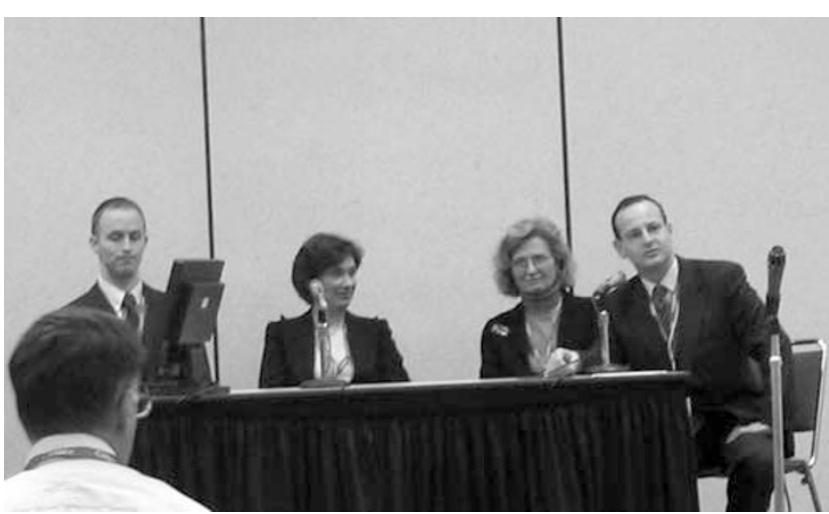

Speakers at IADR Symposium on systematic reviews in dental and craniofacial research (Left to right - Dr's E Manheimer, J Clarkson, K Dickerson and I Needleman).

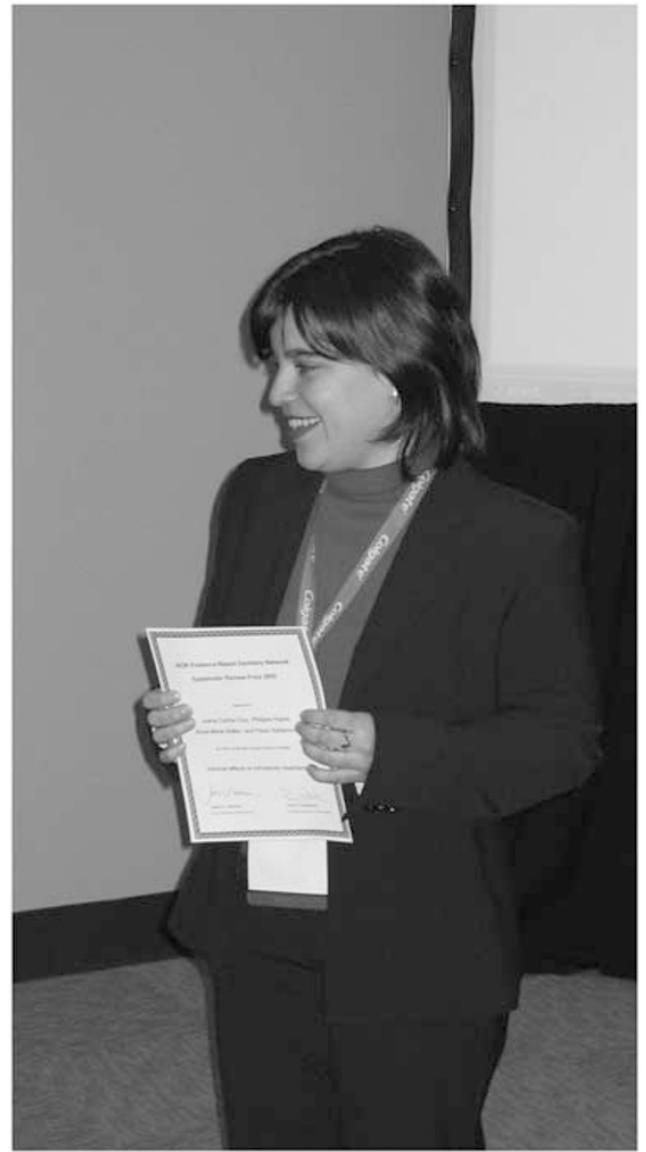

Joana Cunha-Cruz receives systematic review prize.
One of the highlights of the EBNet meeting was the systematic review prize. The prize, which is given to the best systematic review protocol, has been awarded for a several years thanks to generous sponsorship from Colgate, Glaxo SmithKline and Software of Excellence. The judging is conducted by a distinguished panel including Mike Clarke, the Head of the Cochrane Collaboration, Oxford, UK and Jos Kleijnen, the Director of the Centre for Reviews and Dissemination at the University of York, UK. This year's prize was awarded to Joana Cunha-Cruz for her protocol entitled, Adverse effects of orthodontic treatments. The publishers of Evidence-based Dentistry were also pleased to announce that they would be sponsoring the prize for the next 3 years.

Members of IADR can become members of EBNet by subscription as with other groups with IADR.

\section{Medline referencing of EBD}

Regular readers of Evidence-based Dentistry will know that since last year this journal has been referenced on Medline. It has been brought to our attention recently that summaries of original papers presented in the journal are abstracted on Medline in a different manner to other evidence-based journals. We are in discussion with Medline about this and apologies for any confusion caused.

\section{Problems with journals online CPD pages}

Over the past 6-9 months we have been having repeated problems with the online Continuing Professional Development (CPD) pages of the journal. We understand that this is frustrating to those practitioners who wish to use the facility. We are hoping to have resolved the problems with the current hosting arrangements in time for this issue.

Evidence-Based Dentistry (2005) 6, 51-52.

doi:10.1038/sj.ebd.6400327 